Meta

Journal des traducteurs

Translators' Journal

\title{
Le français, langue médicale internationale
}

\section{A. Manuila et A. Rigolot}

Volume 19, numéro 1, mars 1974

La traduction médicale

URI : https://id.erudit.org/iderudit/003555ar

DOI : https://doi.org/10.7202/003555ar

Aller au sommaire du numéro

Éditeur(s)

Les Presses de l'Université de Montréal

ISSN

0026-0452 (imprimé)

1492-1421 (numérique)

Découvrir la revue

Citer cet article

Manuila, A. \& Rigolot, A. (1974). Le français, langue médicale internationale. Meta, 19(1), 3-12. https://doi.org/10.7202/003555ar d'utilisation que vous pouvez consulter en ligne.

https://apropos.erudit.org/fr/usagers/politique-dutilisation/ 


\section{Le français, langue médicale internationale}

\section{LE FRANÇAIS MÉDICAL AU XIX SIÈCLE}

Jusqu'à la Première Guerre mondiale, les francophones disposaient d'un outil remarquable : la langue française. Indépendamment de sa valeur en tant que moyen de communication courante ou d'expression littéraire, philosophique et politique, celle-ci était, sur le plan de la francophonie, un véhicule extrêmement efficace de la pensée scientifique au service de la recherche et de l'enseignement. Mieux encore, en raison même de sa richesse et de sa précision, elle était devenue, grâce à l'intense activité créatrice d'innombrables chercheurs, l'une - sinon la première - des deux grandes langues scientifiques sur le plan à proprement parler international.

Cette prééminence était particulièrement manifeste dans le domaine qui nous intéresse ici : la médecine. Il suffit de rappeler à cet égard l'abondance et la diversité de la bibliographie et surtout de la lexicographie de langue française au $\mathrm{xIX}^{\mathrm{e}}$ siècle. Le nombre et l'ampleur des dictionnaires médicaux parus au cours des quelque cent années qui ont précédé la Première Guerre mondiale sont aujourd'hui pour nous un sujet d'étonnement. Mentionnons, ne serait-ce que pour mémoire, le petit dictionnaire de Capuron (publié en 1806) parce qu'il peut être considéré comme marquant le début de la lexicographie médicale moderne en langue française. Il devait faire l'objet de rééditions, sous les noms de Capuron et Nysten d'abord, sous celui de Nysten seul ensuite. Aussitôt s'ouvrait la série des ouvrages monumentaux avec les dictionnaires d'Allard et coll. (1812) et d'Adelon et coll. (1812 également), ce dernier comptant vingt et un volumes. Ce record devait être battu en 1864 par les dictionnaires de Bernutz et coll. et surtout de Dechambre et coll., le premier avec quarante volumes et le second avec cent volumes. De pareilles entreprises seraient inconcevables de nos jours. Parallètement, des dictionnaires plus modestes continuaient la lignée des Capuron, et le Littré médical, dans ses diverses éditions revues par Robin ou Gilbert, figure encore en bonne place dans la bibliothèque de nombreux praticiens privés. 
Rien de comparable n'existait dans aucune autre langue.

1

Depuis 1918 et surtout depuis 1945, la situation s'est prodigieusement dégradée sur les deux plans précités : celui de la qualité de l'outil mis entre les mains des scientifiques francophones et celui du rôle international du français.

\section{Le français médical actuel dans la francophonie}

Sur le plan de la francophonie, l'outil s'est émoussé pour des raisons diverses, les unes valables pour toutes les langues, les autres plus spécialement propres au français.

Jusqu'au début du $\mathrm{XX}^{\mathrm{e}}$ siècle, le médecin comprenait pratiquement tous les termes du vocabulaire médical. Aujourd'hui, seul le spécialiste connaît vraiment les termes de sa discipline, et encore faut-il faire des réserves à cet égard. On assiste, en effet, à une prolifération de la terminologie dans toutes les sciences, mais peut-être cette explosion terminologique est-elle plus sensible - et plus dangereuse - en médecine.

Ce phénomène a de multiples causes, au premier rang desquelles figure la tendance à l'hyperspécialisation. Si le malade se plaint de plus en plus du fractionnement de la médecine en disciplines souvent cloisonnées, le chercheur et l'enseignant en sont aussi les victimes. En effet, non seulement les connaissances, mais les jargons techniques tendent à se diversifier et parfois même à diverger. Qui plus est, le spécialiste en arrive à être débordé par le foisonnement terminologique de sa discipline. Essayant de renouer avec la tradition lexicographique dont il a été fait mention plus haut, une équipe a entrepris, il y a plusieurs années, d'élaborer un Dictionnaire français de médecine et de biologie ${ }^{1}$. Au cours de la préparation de cet ouvrage, les auteurs ont pris pour base, en matière d'ophtalmologie, le Lexikon Ophtalmologicum en six langues publié sous les auspices du Conseil international d'ophtalmologie. Or, il s'est avéré que les membres mêmes de ce Conseil n'étaient pas en mesure de donner le sens de certains termes du Lexikon. Les enquêtes menées auprès de spécialistes de réputation mondiale restèrent infructueuses. C'est ainsi que les termes en question, ne pouvant être correctement définis, durent être exclus du dictionnaire.

Or, l'approche pluridisciplinaire des problèmes médicaux est, de nos jours, une nécessité de plus en plus impérieuse. Telle question de pédiatrie ne pourra être résolue qu'avec l'appui, non seulement de l'anatomie pathologique, mais encore de l'immunologie, de la biochimie et de la génétique. Malheureusement, la spécialisation croissante des terminologies, qui sont loin d'être toujours concordantes, complique considérablement le problème de la communication.

Cependant, le plus grand facteur de confusion est sans doute l'internationalisation de la médecine. Alors que l'Europe était autrefois le foyer de la recherche

1. A. Manuila, L. Manuila, M. Nicole et $\mathrm{H}$. Lambert, Dictionnaire français de médecine et de biologie, 3 vol. (A-Z), Paris, Masson, 1970-1973; vol. 4 (Annexe) : sous presse. 
médicale, c'est maintenant dans le monde entier que celle-ci s'épanouit. Les congrès internationaux, les échanges de chercheurs, la multiplication des revues scientifiques, par le brassage d'idées qui en résulte, intensifient l'explosion terminologique.

C'est précisément cette explosion qui, pour sensible qu'elle soit dans toutes les langues, menace plus spécialement le français en tant que langue de la francophonie médicale. Ici, une remarque préalable s'impose : toute prise de position dogmatique doit être écartée. Quand un chercheur non francophone propose un concept nouveau, il est normal que ses pairs, dans tous les pays, utilisent au début ses termes mêmes pour éviter les malentendus. En revanche, une fois le concept précisé et confirmé par l'expérience, une terminologie conforme à son génie propre devient indispensable dans chaque langue. Des équivalents doivent être cherchés, même s'ils sont difficiles à trouver. Certes, dans quelques cas, l'adoption du terme étranger peut être inévitable. En général, cependant, une solution est possible : traduction, création d'un néologisme ou simplement naturalisation du terme étranger dans son orthographe et sa prononciation.

Or, nous assistons à une invasion de termes étrangers dans la langue française. Ce sont, d'ailleurs, surtout les termes anglais qui pénètrent dans le français, même pour exprimer des notions fondamentales, voire sans spécificité médicale. Abstraction faite des clearance, stress, stripping, flutter, crossing-over, pace-maker, patchtest, etc., que l'on songe aux feed-back, double-blind test, follow-up, birth-control et planning pour ne citer que quelques exemples. On dirait que le français n'est plus en mesure de lutter contre l'invasion de l'anglais parce qu'il n'arrive plus à suivre le rythme de la création terminologique, et certains vont même jusqu'à accuser la rigidité de la langue. Pour quelle raison le français serait-il soudain frappé de stérilité et ne permettrait-il plus à l'homme contemporain de traduire sa pensée ?

\section{Le français médical, langue internationale}

Cette apparente passivité de la francophonie nous amène tout naturellement à examiner le deuxième fait signalé plus haut : le recul du français en tant que langue internationale. Ce recul est, en effet, pour beaucoup dans la pénétration massive de termes anglais dans notre jargon médical. Il ne faut, d'ailleurs, pas oublier que la défense du français sur le plan international passe par sa consolidation sur le plan de la francophonie.

La régression du français est, certes, due en premier lieu à des facteurs politiques et économiques qui ont affecté notre langue en tant que langue de la diplomatie, du commerce et de la culture. Se rappelle-t-on que le français n'a été admis comme langue officielle de 1'O.N.U., en 1945, qu'à une voix de majorité ? Il est vrai que, dans le domaine politique, la décolonisation lui a donné un regain de vigueur. Qu'on ne s'y trompe pas, cependant : le français, n'est plus que la deuxième langue de travail, présentement sur la défensive devant l'ascension de concurrents sérieux comme l'espagnol, en particulier à l'O.N.U. et dans les institutions spécialisées. D'ailleurs, au point de vue scientifique et par conséquent médical, la décolonisation a, pour l'instant, été d'un faible secours. Les jeunes nations indépendantes, confrontées à d'immenses problèmes de tous ordres, n'ont 
pas encore les ressources nécessaires pour jouer pleinement leur rôle dans la recherche et la communication médicales. En fin de compte, les prodigieux bouleversements survenus depuis 1914 ne pouvaient pas manquer d'exercer une action défavorable sur l'utilisation du français médical dans le monde, puisqu'ils mettaient en cause la vocation internationale du français en général.

Cependant, le grand facteur de régression du français médical est à chercher dans la place comparativement plus modeste qui revient de nos jours à la science francophone par rapport à la science anglophone. Il ne s'agit pas d'incriminer une prétendue stagnation de la recherche francophone. Certes, les effets de deux terribles guerres mondiales ne sauraient, à cet égard, être négligés, mais ils n'ont été que temporaires. En fait, le phénomène qui a radicalement transformé la situation est l'extraordinaire ascension des États-Unis d'Amérique au premier rang des nations. Les pays francophones, compte tenu de leur poids démographique et de leurs disponibilités financières, ne sont pas en mesure de rivaliser avec les ÉtatsUnis appuyés par l'ensemble des pays anglophones. C'est pourquoi l'internationalisation de la médecine joue surtout en leur défaveur. Les médecins, comme les spécialistes des autres branches du savoir, ont plus que jamais besoin de se tenir au courant de ce qui se passe en dehors de leurs frontières nationales. Ils sont contraints de lire des publications en diverses langues, de participer à des congrès internationaux et, plus encore, de faire connaître leurs propres travaux. Or, de nos jours, la masse des revues publiées en anglais est écrasante.

En 1958, la National Library of Medecine, à Washington, a dépouillé quelque 2000 périodiques médicaux mondiaux reçus par elle au cours d'un trimestre. Une étude a démontré que $38,2 \%$ de ces périodiques étaient de langue anglaise contre $10,7 \%$ de langue française. Quant aux articles contenus dans ces publications, $37,2 \%$ étaient rédigés en anglais contre $12,9 \%$ en français ${ }^{2}$. Cette étude est déjà ancienne, mais rien ne permet de penser que le déséquilibre ne se soit pas plutôt aggravé.

Nombreux, en effet, sont les chercheurs qui ont déjà essayé et qui continuent à essayer de lutter contre le courant, mais les difficultés se sont révélées immenses, comme en témoignent ces remarques d'un universitaire : "Parler français pour défendre la culture française est certes un objectif des plus louables, mais ceux qui participent à un congrès international... se trouvent souvent devant l'option suivante : ou bien parler français et n'être compris que d'une faible minorité, ce qui fait tourner court la discussion, ou bien parler anglais... et ouvrir ainsi la voie à une discussion plus étendue et plus animée ${ }^{3}$. 》

Il y a plus grave encore. Combien de chercheurs, fiers du passé scientifique de leur pays et confiants dans l'universalité de leur langue, ont vu leurs observations ou leurs découvertes passer inaperçues en dehors de leur cercle national parce qu'elles avaient été publiées en français ? Et souvent, ces mêmes observations ou

2. Estelle Brodman et Seymour I. Taine, "Current Medical Literature : A Quantitative Survey of Articles and Journals », in : Proceedings of the International Conference on Scientific Information (Washington (D.C.), 16-21 novembre 1958), Washington (D.C.), National Academy of Sciences, 1959 , p. 442.

3. Lettre parue dans le Monde, 14 mars 1965. 
découvertes ont été, quelques années plus tard, présentées comme des nouveautés par d'autres auteurs qui ignoraient, ou feignaient d'ignorer, l'antériorité du chercheur francophone.

Il ne faut donc pas s'étonner de la tendance actuelle à considérer l'anglais comme le véhicule de la pensée scientifique. On voit de plus en plus de chercheurs de tous les pays publier dans des revues de langue anglaise : comment les francophones pourraient-ils échapper à l'entraînement ? Une étude menée il y a quelques années par l'Association des universités partiellement ou entièrement de langue française (A.U.P.E.L.F.) a révélé que jusqu'à $25 \%$ des chercheurs francophones de toutes disciplines préfèrent confier, si possible, leurs articles à des revues de «langue étrangère », expression qui désigne l'anglais dans neuf cas sur dix ${ }^{4}$.

Les effets à long terme de cet état de choses sont extrêmement sérieux. Le recul du français en tant que véhicule international de la recherche médicale n'est pas une pure question de prestige. Sa première grande conséquence est qu'il risque, dans certains domaines, de stériliser la recherche vraiment originale à l'intérieur de la francophonie. Une langue n'est pas un simple instrument; c'est aussi le support de tout un ensemble de notions, de traditions, d'attitudes et de préoccupations, c'est-à-dire d'approches des problèmes qui conditionnent la créativité même du chercheur. Imagine-t-on que la technique psychanalytique serait ce qu'elle est si elle n'avait ses racines dans le substrat collectif de la pensée allemande?

La deuxième grande conséquence est de rendre de plus en plus difficile la transmission du savoir dans le cadre de la francophonie et à l'intérieur même des pays francophones. La pénétration de termes intrus dans la langue française et l'incapacité de les naturaliser et, par là même, d'assimiler la pensée étrangère sont une grave menace pour l'enseignement. Dans beaucoup de cas, les mots anglais, sans référence aucune au lexique national, sont imparfaitement compris. Déjà, les étudiants se plaignent de l'ésotérisme inévitable du jargon médical ${ }^{5}$. Quels ne seront pas les obstacles à l'enseignement dans une décennie ou dans un demi-siècle si la tendance actuelle se maintient, compte tenu du rythme de l'innovation scientifique. Et si l'enseignement pâtit, n'est-ce pas la recherche qui ultérieurement en fera les frais?

Face à l'avenir : redonner au français médical une nouvelle vitalité

La question qui se pose est évidente : que peut-on faire devant le monopole grandissant de l'anglais et, plus particulièrement, que peuvent faire les traducteurs ?

Une remarque préliminaire s'impose à ce sujet : les traducteurs ne se limitent plus aux seuls traducteurs professionnels. Ce sont, tout d'abord, les chercheurs et les enseignants qui interviennent dans le processus de traduction. Leur nombre a

4. Voir l'article de J.L. Lavallard dans le Bulletin de traduction de la Commission des Communautés européennes, avril 1970.

5. Une communication intéressante a été présentée sur ce point par C. Rouiller à la Conférencetable ronde sur la terminologie médicale qui s'est tenue à la Maison de l'U.N.E.S.C.O. (Paris), le 15 décembre 1972 (sous presse). 
décuplé et les découvertes s'intensifient; comme nous l'avons dit plus haut, toutes les disciplines font de plus en plus appel à des méthodes empruntées à d'autres sciences (statistique, informatique, etc.). Il en résulte une injection croissante de termes nouveaux dans le langage médical, dont beaucoup sont d'origine étrangère. Le chercheur francophone doit donc leur trouver des équivalents, les assimiler ou les adopter tels quels. Qu'il le veuille ou non, l'homme de science est sans cesse confronté à des problèmes de traduction.

Toutefois - et c'est là un phénomène entièrement nouveau - le langage médical n'est plus l'apanage exclusif des médecins. Nous sommes entrés dans l'ère de la communication accélérée et généralisée. Les hommes de science ont toujours entretenu un dialogue par-dessus les frontières, mais aujourd'hui ils ont de plus en plus besoin «pour dialoguer » de nombreux professionnels de l'information. Ces professionnels - traducteurs, rédacteurs, personnel technique des revues médicales, "abstracteurs », interprètes, voire journalistes - sont aussi confrontés aux problèmes de langage et de traduction. Très souvent ils travaillent dans la hâte. Gros consommateurs de terminologie médicale, ils n'hésiteront pas à créer des vocables s'ils ne les ont pas sous la main au moment voulu, et leur influence sur le lexique médical ne peut aller qu'en s'accroissant avec les années.

Ce sont toutes les personnes énumérées ci-dessus qui ont en main l'avenir du français médical. Il leur faut donc se pénétrer de leurs responsabilités. La langue est un héritage dont nous sommes redevables à nos prédécesseurs, mais c'est aussi une richesse dont nous sommes comptables vis-à-vis des générations futures. Enrichir le français pour lui permettre de suivre la marche de la science est, par conséquent, le premier devoir. Tant que le rythme de l'innovation était lent, le problème n'était pas aigu. Le chercheur qui, obéissant à la facilité ou écoutant les conseils du snobisme, introduisait un terme étranger dans le lexique — rash, flutter, etc. - ne causait pas de dégâts sensibles au français. Les autres langues étaient, d'ailleurs, logées à la même enseigne. Aujourd'hui, en revanche, c'est une invasion qui nous menace, et ce phénomène n'en est qu'à ses débuts. Que deviendra le langage dans un proche avenir si l'on n'y prend garde? Trop d'hommes de science affichent un scepticisme facile à cet égard, parce qu'ils refusent de regarder devant eux. Une campagne menée dans un esprit pragmatique pour la mise à jour continue du français est donc indispensable. Ce qu'il faut, c'est redonner au français médical le dynamisme créateur qui caractérise la langue anglaise, notamment telle qu'elle est utilisée aux États-Unis d'Amérique.

Or, des efforts dans ce sens, sporadiques d'abord, plus vigoureux et mieux coordonnés maintenant, ont été entrepris depuis plusieurs années. Ils commencent à porter leurs fruits, mais ils sont mal connus. La campagne d'enrichissement du français doit donc s'accompagner d'une campagne d'information. Ceux qui ont en main la responsabilité de la langue médicale - médecins et non-médecins ignorent trop souvent ce qui se fait et où ils pourraient trouver les renseignements et les appuis dont ils ont besoin. Un exposé des tentatives en cours pour infuser un renouveau de vitalité au français médical ne sera peut-être pas inutile dans ce contexte. 


\section{Tableau des efforts entrepris}

En premier lieu, le français ne peut que bénéficier de l'action menée pour normaliser les terminologies techniques sur le plan international. Le C.I.O.M.S. (Conseil des organisations internationales des sciences médicales), avec l'aide de l'O.M.S., s'est attelé à cette lourde tâche dans le domaine de la nosologie. Comme l'explique son secrétaire exécutif, le $D^{x} S$. Btesh : " l'idée est de tâcher d'obtenir pour chaque maladie un consensus sur la dénomination à préconiser ${ }^{6} \gg$. Dans chaque cas, il s'agit de fixer une dénomination dans trois langues : l'anglais, l'espagnol et le français. Dans un premier temps, des listes de termes doivent être publiées sous le titre de «Nomenclature internationale provisoire » et largement diffusées. À l'heure actuelle, trois listes ont été terminées et ont paru ou vont paraître. Dans plusieurs autres domaines, d'ailleurs, des efforts plus ou moins similaires ont été accomplis ou sont en voie de l'être pour unifier les nomenclatures dans une ou plusieurs langues. Citons la Parisiensis nomina anatomica, les nomenclatures bactériologiques, zoologiques et botaniques déjà publiées, les travaux de l'Union internationale de chimie pure et appliquée et de l'Organisation internationale de standardisation, la nomenclature histologique en cours de préparation, etc. Ces entreprises témoignent de l'intérêt que suscitent dans le monde les questions de terminologie.

Sur le plan qui nous concerne plus directement ici, celui de la francophonie, l'élan est aussi donné et de nombreux organismes sont au travail. Le tableau ci-joint donne une idée générale des initiatives qui ont été prises en France même pour défendre et enrichir le langage scientifique sous tous ses aspects. En ce qui concerne la médecine et les sciences apparentées, notons que l'Académie nationale de médecine et l'Académie des sciences ont, l'une et l'autre, créé leur propre Commission de terminologie et, tout récemment, le ministère de la Santé a suivi leur exemple. Le Comité d'étude des termes médicaux français, constitué à l'image du Comité d'étude des termes techniques français qui vient de faire paraître un ouvrage remarquable, appelé à servir d'exemple et d'encouragement ${ }^{7}$, s'emploie, lui aussi, sous la présidence du professeur Maurice Lamy et l'impulsion dynamique de ses ancien et nouveau secrétaires, le $\mathrm{D}^{\mathrm{r}}$ Daniel Eyraud et le professeur Jean-Charles Sournia, à lutter contre la dégradation du vocabulaire médical et pour son élargissement. C'est ainsi qu'il a publié deux «Tables d'anglicismes » à proscrire, en donnant des séries d'équivalents conseillés. Il faut, à ce propos, saluer la publication au Journal officiel de la République française de listes de termes techniques dont l'usage par les administrations publiques est soit obligatoire, soit recommandé, en vue d'éliminer l'emploi abusif de mots d'origine étrangère ${ }^{8}$.

Il n'est que juste de rappeler ici le remarquable travail accompli depuis plusieurs années par le Comité canadien d'étude des termes de médecine qu'anime

6. Communication adressée à la Conférence-table ronde sur la terminologie médicale, organisée à la Maison de l'U.N.E.S.C.O. (Paris), le 15 décembre 1972 (sous presse).

7. Termes techniques français, établi par le Comité d'étude des termes techniques français, Paris, Hermann, 1972.

8. Une première série de termes a paru dans le Journal officiel, 18 janvier 1973. Elle porte sur diverses sciences et techniques, mais non sur la médecine. Toutefois des listes ultérieures seront consacrées à la terminologie médicale. 


\section{TABLEAU 1}

Relation entre les principaux o; ganismes français de défense et d'enrichissement de la langue

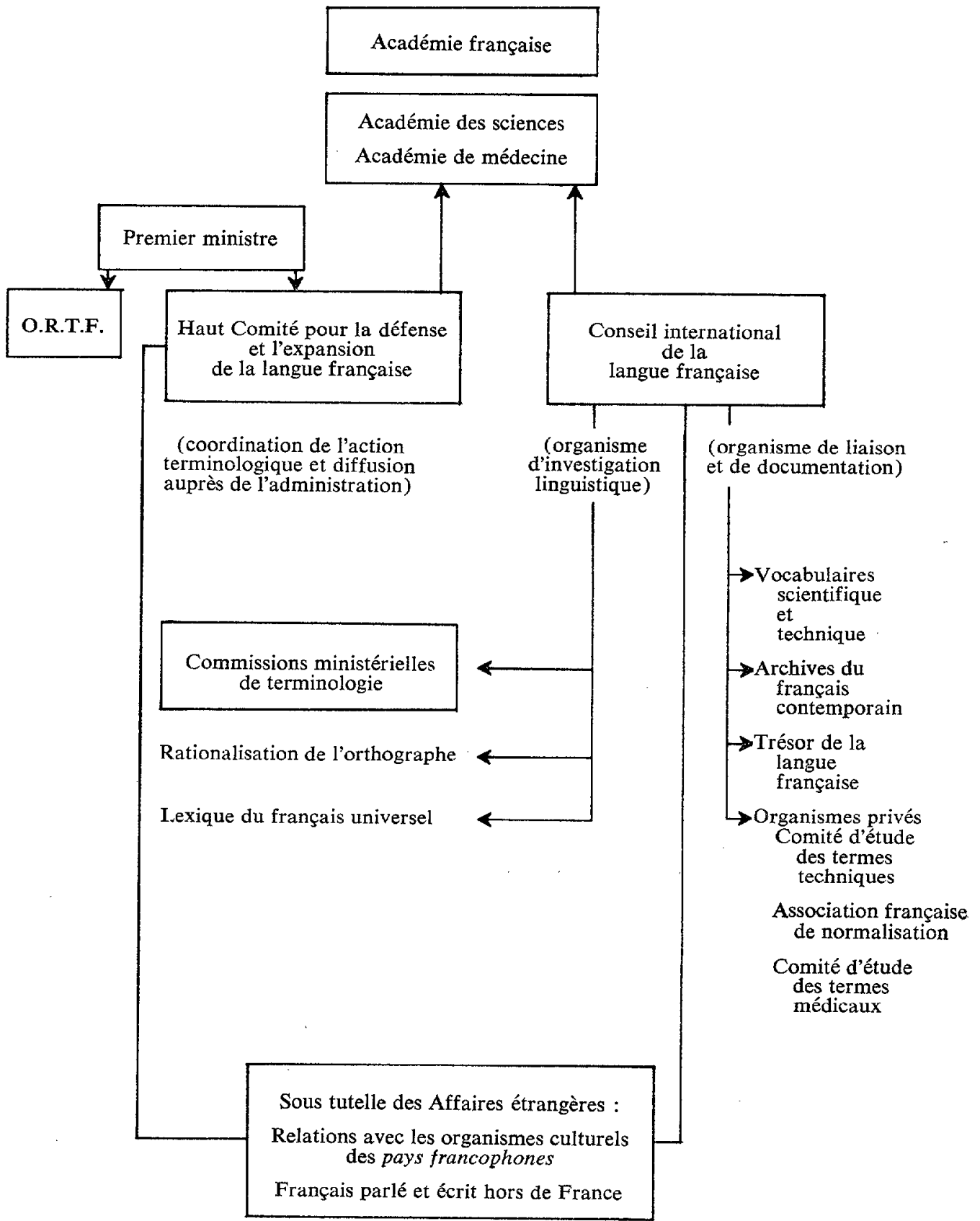


le $\mathrm{D}^{\mathrm{r}}$ Boulay. En effet, le mouvement pour le renouveau du français médical ne se limite pas à la France seule. Nous n'en voulons pour preuve que l'initiative prise sous l'impulsion du professeur Evrard, de Liège, lors du premier Congrès des anesthésistes-réanimateurs d'expression française qui s'est tenu à Versailles en 1966 , de réserver une séance aux problèmes de terminologie ${ }^{9}$. Cette expérience pourrait sans nul doute être étendue à d'autres disciplines.

Tout ce bouillonnement d'activités aboutit peu à peu à la création des termes nécessaires au langage pour suivre l'évolution de la médecine. Simultanément, des travailleurs individuels se sentent encouragés à dresser des glossaires et des dictionnaires plus ou moins spécialisés. Or, les dictionnaires (unilingues, bilingues ou plurilingues) sont une source d'information fondamentale pour ceux qui interviennent dans le circuit de la communication. Malheureusement, ces sources d'information, surtout lorsqu'elles sont très spécialisées, sont trop souvent mal connues et difficiles d'accès. C'est dire tout l'intérêt que revêt la liste d'ouvrages de référence que M. van Hoof a établie pour le présent numéro de Meta.

\section{CONCLUSION}

Le français médical a donc commencé à remonter la pente. Toutefois, trop nombreux sont encore les sceptiques et les indifférents. Il est indispensable que la plus grande publicité soit donnée à l'action déjà engagée pour insuffler une nouvelle vigueur à la langue médicale française. À cet égard, les revues, spécialisées ou non, pourraient jouer un rôle capital en traitant plus largement des problèmes de terminologie et en rendant compte régulièrement des décisions et recommandations formulées par les organismes compétents. Dans la partie qui s'engage, le plus important est peut-être de faire prendre conscience à tous les intéressés du péril qui menace la langue et des moyens de défense à mettre en œuvre.

Les efforts entrepris ont aussi besoin d'être intensifiés et rendus plus efficaces. Les organismes qui ont commencé à agir sont le plus souvent bénévoles, imparfaitement outillés et dépourvus de ressources financières et autres. Il nous paraîtrait souhaitable que les gouvernements responsables leur apportent de plus grands encouragements et des appuis plus substantiels. À l'heure actuelle, en effet, il ne suffit pas de permettre au français de suivre l'évolution terminologique médicale : il faut l'aider à rattraper un retard.

Il nous semble aussi que cette tâche doit être abordée dans un esprit largement international. Aussi, souhaiterions-nous que, dans les organismes terminologiques, les médecins ne soient pas seuls à être représentés. Ceux que nous avons appelés les professionnels de la communication - notamment les traducteurs pourraient peut-être y faire prévaloir des conceptions à la fois plus pratiques et plus ouvertes.

Une mise en garde s'impose toutefois : il ne faut pas confondre, comme on le fait souvent, promotion de la recherche des pays francophones et défense du

9. Comptes rendus du $1^{\text {er }}$ Congrès des anesthésistes-réanimateurs d'expression française (16 Congrès national français d'anesthésie-réanimation, Versailles, 3, 4, 5 et 6 juin 1966). 
français. La recherche des pays francophones est une réalité qui demande une légitime consécration, mais le français n'est qu'un des instruments qui peuvent l'aider à se faire connaître et reconnaître. La recherche germanique, par exemple, a pu acquérir dès la fin du Xrx ${ }^{e}$ siècle une réputation mondiale sans que l'allemand eût été un véhicule international.

Il faut, d'ailleurs, s'entendre sur la notion de langue internationale. Dans le contexte historique actuel, le français ne saurait prétendre faire jeu égal avec l'anglais comme langue médicale internationale. En revanche, il peut - et doit, si les francophones comprennent l'importance du problème - demeurer la deuxième langue médicale du monde. Face au dynamisme de l'anglais (et surtout dẹ sa variante américaine) il est vital que le français retrouve la souplesse et les possibilités d'enrichissement qu'il a perdues entre les deux guerres. Mais cela, c'est l'affaire de la francophonie. Espérons qu'elle en prendra conscience : l'enjeu en vaut la peine.

A. Manuila et A. Rigolot 